\title{
Beauty Photoproduction at ZEUS
}

\author{
Ursula Samson on behalf of the ZEUS Collaboration \\ Physikalisches Institut Universität Bonn \\ Nussallee 12, 53115 Bonn, Germany
}

\begin{abstract}
Beauty production in the photoproduction regime $\left(Q^{2}<1 \mathrm{GeV}^{2}\right)$ using events with semileptonic beauty decays has been measured with the ZEUS detector. Three different analyses measuring beauty production are presented here. The first analysis is a preliminary result using $124 \mathrm{pb}^{-1}$ data taken in 2005, while the second and third analysis are final results using $120 \mathrm{pb}^{-1}$ data taken between 1996 and 2000. Differential cross-sections are extracted, as well as the total beauty production cross-section. The measurements are compared to each other and to previous results from the ZEUS and $\mathrm{H} 1$ collaborations as a function of the transverse momentum of the beauty quark.
\end{abstract}

\section{Introduction}

Beauty production at HERA is an important tool to investigate our present understanding of the theory of Quantum Chromo-Dynamics (QCD). On the one hand the large b mass, taken as the hard scale, ensures that the cross-sections are always perturbatively calculable. On the other hand, the simultaneous presence of competing hard scales, in the photoproduction case mainly the transverse momentum $\left(p_{t}\right)$ of the heavy quark, induces additional theoretical uncertainties due to terms in the perturbative expansion which depend logarithmically on the ratio of these scales. Results on three different measurements in the photoproduction regime using the ZEUS detector are presented here [1]. A detailed description of the ZEUS detector can be found elsewhere [2].

\section{Theoretical predictions}

The measurements are compared to leading order (LO) plus parton shower (PS) Monte Carlo predictions. In the case of the dijet analyses the PYTHIA Monte Carlo program was used, while in the dimuon analysis a mixture of PYTHIA and RAPGAP was used in order to take into account the DIS contribution in this analysis. For quantitative comparisons QCD next-to-leading order (NLO) predictions in the massive approach [3] are used. The photoproduction cross-section is calculated using the Weizsäcker-Williams approximation (WWA) with an effective $Q_{\max }^{2}$ cutoff of $1 \mathrm{GeV}^{2}$. For the analysis using a dimuon double tag the cutoff was raised to $25 \mathrm{GeV}^{2}$ in order to include the DIS contribution to the crosssection. In the case of the dijet analyses the factorisation and renormalisation scales are set to $\mu=\mu_{0}=\sqrt{p_{t}^{2}+m_{b}^{2}}$, while the scales are set to $\mu=\mu_{0} / 2$, as there are hints that this might be a better choice[4].

\section{Measurement of beauty photoproduction at HERA II}

A sample of photoproduction events with two jets and a muon in the final state was selected from $e p$ collisions collected during 2004 and 2005, corresponding to an integrated luminosity of $\mathcal{L}=124 \mathrm{pb}^{-1}$. The jets were reconstructed using the KTCLUS algorithm in the longitudinal invariant mode in the $E$-recombination scheme. At least two jets with $\left|\eta^{j e t}\right|<2.5$ 
and $p_{T}^{\text {jet1(2) }}>7(6) \mathrm{GeV}$ were required. At least one jet was required to contain a muon candidate of $p_{T}^{\mu}>2.5 \mathrm{GeV}$ in a pseudorapidity range of $-1.6<\eta<2.3$. Standard cuts and requirements were applied to reject DIS events. After this selection 7424 muons were left. The heavy flavour content of the selected sample was determined by means of the transverse momentum of the muon with respect to the jet axis, $p_{T}^{r e l}$, and the impact parameter of the muon track calculated as the closest distance of approach of the muon with respect to the beamspot, $\delta$. While the $p_{T}^{r e l}$ variable separates well beauty from charm and light flavour, the impact parameter separates the beauty and charm contribution from the light flavour. To extract the fractions of $b, c$ and $l f$ in the sample a binned $\chi^{2}$ fit of the two-dimensional distribution of $p_{T}^{r e l}$ versus $\delta$ was performed by adjusting the relative normalisation of the three MC samples to reproduce the data. Using the fit result, a total visible cross-section was obtained and compared to QCD NLO predictions:

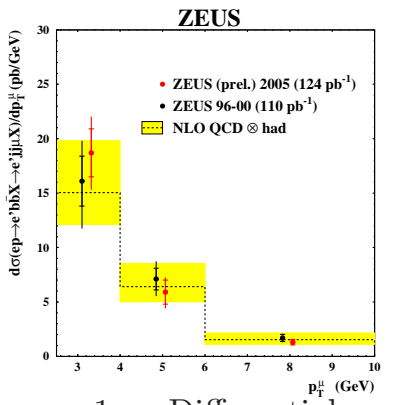

Figure 1: Differential crosssection as a function of $p_{T}^{\mu}$

$$
\sigma_{b \bar{b}}^{v i s}=46.8 \pm 4.0(\text { stat. })_{-7.2}^{+6.1} \text { (syst.) } \mathrm{pb}, \quad \sigma_{N L O}^{v i s}=41.5_{-8.9}^{+13.9} \mathrm{pb}
$$

for the process $e p \rightarrow e^{\prime} b \bar{b} X \rightarrow e^{\prime} j j \mu X^{\prime}$ in the kinematic range $Q^{2}<1 \mathrm{GeV}, 0.2<y<$ $0.8, p_{T}^{\text {jet } 1(2)}>7(6) \mathrm{GeV},\left|\eta^{j e t}\right|<2.5, p_{T}^{\mu}>2.5 \mathrm{GeV}$ and $-1.6<\eta^{\mu}<2.3$. The cross-section was found in agreement with the corresponding QCD NLO prediction. Differential crosssections were measured in the same kinematic range as a function of the muon transverse momentum $d \sigma / d p_{T}^{\mu}$ and the muon pseudorapidity, $d \sigma / d \eta^{\mu}$. The beauty fraction was determined independently for each cross-section bin. The results [5] are compared and found in good agreement to a measurement of the ZEUS HERA-I data [6] and to QCD NLO predictions computed with the FMNR program. The beauty cross-sections as a function of $p_{T}^{\mu}$ is shown in fig. 1. For the measurement with the HERA-I data, only the $p_{T}^{r e l}$ variable could be used to discriminate between beauty and background. The relative fraction of charm and light flavour were therefore fixed in this analysis.

\section{Beauty photoproduction using decays into electrons at HERA}

Similar to the analysis presented in the previous section this analysis [7] is based on a dijet plus lepton selection, but in this case at least one $b$ quark decays semileptonically into an electron: $e p \rightarrow e^{\prime} b \bar{b} X \rightarrow e^{\prime} j j e_{s l} X^{\prime}$. The identification of electrons close to a jet is more difficult than the identification of muons, but electrons can be identified down to lower momenta. At least two jets with $\left|\eta^{\text {jet }}\right|<2.5$ and $E_{T}^{j e t 1(2)}>7(6) \mathrm{GeV}$ were required, reconstructed using the KTCLUS algorithm in the E-recombination scheme. At least one jet was required to contain an electron candidate of $p_{T}^{e}>0.9 \mathrm{GeV}$ in a pseudorapidity range of $|\eta|<1.5$. Standard cuts and requirements were applied to reject DIS events. Beauty was extracted using a likelihood ratio test of five different input variables: $d E / d x$, the average energy loss per unit length of the track in the central tracking device; $E_{E M C} / E_{C A L}$, the fraction of the calorimeter energy, $E_{C A L}$, deposited in the the electro-magnetic part, $E_{E M C} ; E_{C A L} / p_{\text {track }}$, the energy of the EFO taken from the calorimeter information divided by the track momentum; $p_{T}^{r e l}$, the transverse momentum of the electron with respect to the jet axis and $\Delta \phi$; the azimuthal angle between the electron candidate and the missing transverse momentum vector. 
While the first three variables are for electron identification, the last two discriminate between semileptonic beauty and charm and background events. The test function $T$ is calculated for the electron hypothesis from semileptonic beauty decays and the distribution of $-2 \ln T$ used to determine the fractions of beauty and charm from semileptonic electron decays and background in a three parameter maximum likelihood fit of the different Monte Carlo samples to the data in a range of $-2 \ln T<10$. The total visible cross-section for beauty and charm in the kinematic range of $Q^{2}<1 \mathrm{GeV}^{2}, 0.2<y<$ $0.8, E_{T}^{\text {jet1(2) }}>7(6) \mathrm{GeV},\left|\eta^{\text {jet }}\right|<2.5, p_{T}^{e}>0.9 \mathrm{GeV}$ and $\left|\eta^{e}<1.5\right|$ is

$$
\sigma_{b}^{v i s}=125 \pm 11(\text { stat. })_{-11}^{+10}(\text { syst. }) \mathrm{pb}, \quad \sigma_{c}^{v i s}=278 \pm 33(\text { stat. })_{-24}^{+48}(\text { syst. }) \mathrm{pb} .
$$

The cross-sections were found in agreement with the corresponding QCD NLO predictions:

$$
\sigma_{b}^{N L O}=88.5_{-13}^{+22} \mathrm{pb}, \quad \sigma_{c}^{N L O}=380_{-110}^{+170} \mathrm{pb} .
$$

Differential cross-sections have been measured as a function of $p_{T}^{e}, \eta^{e}, x_{\gamma}^{j}$ et, $E_{T}^{j e t 1}, \eta^{j e t 1}, E_{T}^{e}$ jet , and $\eta^{e}$ jet for beauty and charm. All measured cross-sections agree within the uncertainties with the QCD NLO predictions. The beauty cross-sections as a function of $p_{T}^{e}$ is shown in fig. 2 .

\section{Measurement of total and differential cross-sections for beauty production from dimuon events at HERA}

The third analysis [8] is based on a dimuon double tag. No photoproduction selection is applied, the contribution with DIS events is about $15 \%$. The event selection is mainly based on the requirement of two good muon candidates with a transverse momentum of $p_{T}^{\mu}>1.5 \mathrm{GeV}$ and of $p_{T}^{\mu}>0.75 \mathrm{GeV}$ for high quality muons. The selected events can be classified in four classes of like-sign and unlike-sign combinations with a high $\left(m_{\mu \mu}>4 \mathrm{GeV}\right)$ or a low $\left(m_{\mu \mu}<4 \mathrm{GeV}\right)$ dimuon mass. 2 muons originating from the same beauty quark through the sequential decay $b \rightarrow c \mu X \rightarrow s \mu \mu X^{\prime}$ lead to unlike-sign combinations with a low dimuon mass, while muons from different b quarks

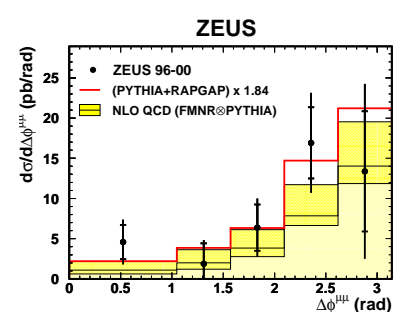

Figure 3: Differential crosssection as a function of $\Delta \phi^{\mu \mu}$, $\left(m^{\mu \mu}>3.25 \mathrm{GeV}\right)$ lead to like-sign and unlike-sign combinations with a high dimuon mass. An important background source comes from primary charm-quark pair production, where both quarks decay semileptonically into a muon. These can only lead to unlike-sign contributions. Other background leading to unlike-sign combinations include heavy quarkonium (VM) and Bethe-Heitler (BH) processes. These contributions can be very well estimated, as they are not directly accompanied by hadronic activity. Background from false muons i.e. from decays in flight or misidentified hadrons are almost charge uncorrelated, thus should give equal contributions to the like-sign and unlike-sign combinations. 
The number of beauty events $N_{b \bar{b} \rightarrow \mu \mu}$ can therefore be measured from the difference of likesign and unlike-sign combinations. Using this method to determine the beauty content, the total $e p$ cross-section for $b \bar{b}$ production has been measured and found in agreement with the QCD NLO prediction within errors:

$$
\sigma^{\text {tot }}=13.9 \pm 1.5(\text { stat. })_{-4.3}^{+4.0}(\text { syst. }) \mathrm{nb}, \quad \sigma_{N L O}=7.8_{-2.3}^{+4.9} \mathrm{nb} .
$$

Differential cross-sections have been measured as a function of the muon variables and the azimuthal angle $\Delta \phi^{\mu \mu}$ between the two muons, measuring correlations between the two beauty quarks, see fig. 3. All differential cross-sections are in agreement with the theoretical predictions.

\section{Conclusions}

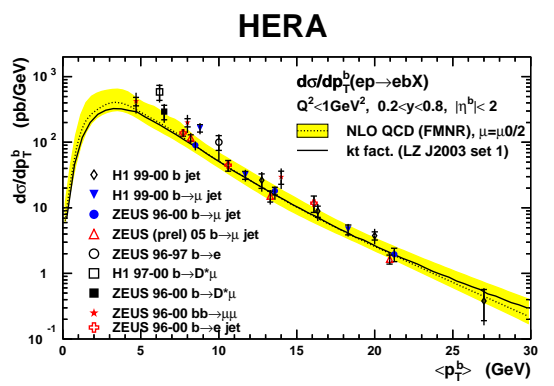

Figure 4: Differential cross-section as a function of $p_{T}^{b}$.

For each analysis the cross-section was determined as a function of the transverse momentum of the beauty quark and compared to previous measurements as well as to QCD NLO predictions, see fig. 4. While older measurements tend to be higher than the predictions, the measurements presented here are all in agreement with the predictions.

\section{References}

[1] Slides:

http://indico.cern.ch/getfile.py/access?contribid=231\&sessionid=14\&resid=0\&materialid=slides\&confid=24657.

[2] The ZEUS detector. Status Report (unpublished), DESY, 1993.

[3] S. Frixione et al. Heavy-quark correlations in photon-hadron collisions. Nucl. Phys., B 412:225, 1994.

[4] A. Geiser. Review of beauty production at hera and elsewhere.

[5] S. Chekanov et al. ZEUS Coll. Measurement of beauty photoproduction at HERA II, 2007.

[6] S. Chekanov et al. ZEUS Coll. Bottom photoproduction measured using decays into muons in dijet events in $e p$ collisions at $\sqrt{s}=318$ gev. Phys. Rev., D 70:12008, 2004.

[7] S. Chekanov et al. ZEUS Coll. Beauty photoproduction using decays into electrons at HERA. DESY08-056, to be published in Physical Rewiew D, 2008.

[8] I. Bloch. Measurement of beauty production from dimuon events at HERA ZEUS. DESY-THESIS-2005034. 